\title{
FIXED-POINT-FREE EMBEDDINGS OF GRAPHS IN THEIR COMPLEMENTS
}

\author{
SEYMOUR SCHUSTER \\ Carleton College \\ Northfleld, Minnesota 55057 U.S.A.
}

(Received January 20, 1978)

ABSTRACT. The following is proved: If $G$ is a labeled $(p, p-2)$ graph where $p \geq 2$, then there exists an isomorphic embedding $\phi$ of $G$ in its complement $\bar{G}$ such that $\phi$ has no fixed vertices. The extension to $(p, p-1)$ graphs is also considered.

KEY WORDS AND PHRASES. Labeled graph, complement, and embedding. AMS(MOS) SUBJECT CLASSIFICATION (2970) CODE. Primary $05 C Z 0$.

If $G$ is a graph, then $V(G)$ and $E(G)$ will denote its vertex set and edge set, respectively. Further, $G$ is called a $(p, q)$ graph if $|V(G)|=p$ and $|E(G)|=q$. An embedding of $G$ in a graph $H$ is an isomorphic mapping of $G$ into $\mathrm{H}$; in other words, there exists an embedding of $\mathrm{G}$ in $\mathrm{H}$ if $\mathrm{H}$ contains a subgraph which is an isomorphic copy of $G$.

The fact that every ( $p, p-2)$ graph $G$ can be embedded in its complement $\bar{G}$ was proven, independently, in [1], [2], and [4]. In the present paper, we establish a strengthened version of this result and also consider exten- 
sions. First of all, we assume that $G$ is labeled; then it becomes meaningful to ask whether the embedding has fixed vertices. This question has pertinence in the study of embedding $(p, p-1)$ graphs in their complements. Indeed, the theorem we prove here serves as a useful tool in characterizing those (p,p-1) graphs which are embeddable in their complements (see [3]).

THEOREM 1. If $G$ is a labeled $(p, p-2)$ graph where $p \geq 2$, then there exists an isomorphic embedding $\phi$ of $G$ in $\bar{G}$ such that $\phi$ has no fixed vertices.

PROOF. The proof is by induction.

The theorem is clearly true for $p=2$ and 3 . We assume that it holds, also, for all $(p, p-2)$ graphs where $p<k$ and $k \geq 4$, and we consider $G$ to be an arbitrary $(k, k-2)$ graph. (N.B. This induction hypothesis implies that the theorem also holds for all ( $p, p-n)$ graphs, where $n>2, p<k$ and $k \geq 4$.)

First, we suppose that $G$ has an isolated vertex $v$. Since $G$ has $k-2$ edges, it must possess a vertex $u$ of degree greater than one. Then $G_{1}=$ $G-\{u, v\}$ is a $(k-2, k-n)$ graph, with $n \geq 4$, so the induction hypothesis guarantees the existence of an embedding $\phi: G_{1} \rightarrow \bar{G}_{1}$ which maps no vertex of $G_{1}$ onto itself. This embedding can be extended to an embedding of $G$ in $\bar{G}$ by defining $\phi(u)=v$ and $\phi(v)=u$. It is clear that this extension, also, has no fixed vertices.

Henceforth, we assume that $G$ has no isolated vertices.

Since every cyclic component having $r$ vertices has at least $r$ edges, the components of $G$ must include at least two non-trivial trees $T_{1}$ and $T_{2}$. If one of these trees, say $T_{1}$, is of order two, we write $V\left(T_{1}\right)=$ $\left\{v_{1}, v_{2}\right\}$ and consider $G_{2}=G-v_{2}$, which is a $(k-1, k-3)$ graph. The induction hypothesis guarantees a fixed-point-free embedding $\sigma$ of $G_{2}$ in $\bar{G}_{2}$. We define $\phi: G \rightarrow \bar{G}$ as follows:

$$
\begin{gathered}
\phi\left(v_{1}\right)=v_{2}, \phi\left(v_{2}\right)=\sigma\left(v_{1}\right) \text {, and } \\
\phi(v)=\sigma(v) \text { for all } v \varepsilon v(G) \text {, and } v \neq v_{1} .
\end{gathered}
$$


With this definition, it is easy to see that $\phi$ is a fixed-point-free embedding of $G$ in $\bar{G}$.

If neither $T_{1}$ nor $T_{2}$ is of order two, we form the graph $G_{3}=G-T_{1}$. Let $\mathrm{x} \in \mathrm{V}\left(\mathrm{T}_{1}\right)$ be a vertex of degree at least two and $\mathrm{y} \varepsilon \mathrm{V}\left(\mathrm{G}_{3}\right)$ also of degree at least two. Then the subgraphs $T_{1}-x$ and $G_{3}-y$ both satisfy the induction hypothesis. Let $\sigma: \mathrm{T}_{1}-\mathrm{x} \rightarrow \overline{\mathrm{T}_{1}-\mathrm{x}}$ and $\beta: \mathrm{G}_{3}-\mathrm{y} \rightarrow \overline{\mathrm{G}_{3}-\mathrm{y}}$ be fixed-pointfree embeddings. We define $\phi: G \rightarrow \bar{G}$ as follows:

$$
\begin{aligned}
\phi(\mathrm{x})= & \mathrm{y}, \phi(\mathrm{y})=\mathrm{x}, \phi(\mathrm{v})=\sigma(\mathrm{v}) \text { for } \mathrm{all} \mathrm{v} \varepsilon \mathrm{V}\left(\mathrm{T}_{1}-\mathrm{x}\right) \\
& \text { and } \phi(\mathrm{v})=\beta(\mathrm{v}) \text { for all } \mathrm{v} \varepsilon \mathrm{V}\left(\mathrm{G}_{3}-\mathrm{y}\right) .
\end{aligned}
$$

This produces a fixed-point-free embedding of $G$ in its complement, thus completing the proof of the theorem.

The foregoing result is "best possible" in the sense that there exist $(\mathrm{p}, \mathrm{p}-1)$ graphs which are embeddable in their complements, but which cannot be so embedded without fixed vertices. Two simple examples arise in considering the disjoint union of a small star and a 3-cycle: viz., $K_{1,2} \cup \mathrm{C}_{3}$ and $\mathrm{K}_{1,3} \cup \mathrm{C}_{3}$. However, it is interesting to note that all other $(\mathrm{p}, \mathrm{p}-1)$ graphs that are contained in their complements can be embedded without fixed vertices. By slight modifications of the arguments in [3], one can prove the following.

THEOREM 2. Let $G$ be a labeled ( $p, p-1)$ graph such that (a) $G$ is embeddable in its complement and (b) $\mathrm{G} \neq \mathrm{K}_{1,2} \cup \mathrm{C}_{3}$ and $\mathrm{G} \neq \mathrm{K}_{1,3} \cup \mathrm{C}_{3}$. Then there exists a fixed-point-free embedding of $G$ in $\bar{G}$.

This result can be stated more explicitly by defining the class $\Omega$ of graphs to consist of $\mathrm{K}_{1} \cup \mathrm{C}_{4}, \mathrm{~K}_{1} \cup 2 \mathrm{C}_{3}, \mathrm{~K}_{1, \mathrm{p}-1}$, and $\mathrm{K}_{1, \mathrm{n}} \cup \mathrm{C}_{3}$ for $\mathrm{n} \geq 0$ (assuming the convention that $K_{1,0}=K_{1}$ ). Then Theorem 2 says: If $G$ be any labeled $(p, p-1)$ graph, then there is a fixed-point-free embedding of $G$ in $\bar{G}$ if and only if $G \notin \Omega$. 
While Theorem 1 plays a role in the proof of further embedding theorems, Theorem 2 does not enjoy such significance; it seems not to possess anything beyond intrinsic interest. For this reason, primarily, we haven't given more attention to the proof of Theorem 2. The interested reader should find little difficulty in constructing the fixed-point-free embeddings of ( $p, p-1)$ graphs from the proofs provided in [3].

ACKNOWLEDGEMENT. The author expresses his appreciation to the Mellon Foundation for a grant which partially supported his research during 1976-78.

\section{REFERENCES}

1. Bollobhs, B. and S. E. Eldridge. Packings of Graphs and Applications to Computational Complexity, Proceedings of the Fifth British Combinatorial Conference (Aberdeen, 1975), Congressus Numerantium XV, Utilitas Mathematica Publishing.

2. Burns, D. and S. Schuster. Every $(\mathrm{p}, \mathrm{p}-2)$ Graph is Contained in its Complement, Journal of Graph Theory 1 (1977) 277-279.

3. Burns, D. and S. Schuster. Embedding ( $p, p-1)$ Graphs in Their Complements, Israel Journal of Mathematics (to appear).

4. Sauer, N. and J. Spencer. Edge Disjoint Placement of Graphs, Journal of Combinatorial Theory (B) (to appear). 


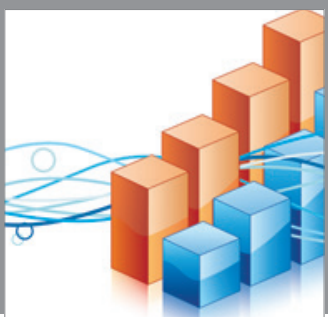

Advances in

Operations Research

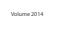

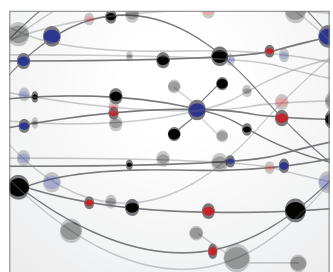

\section{The Scientific} World Journal
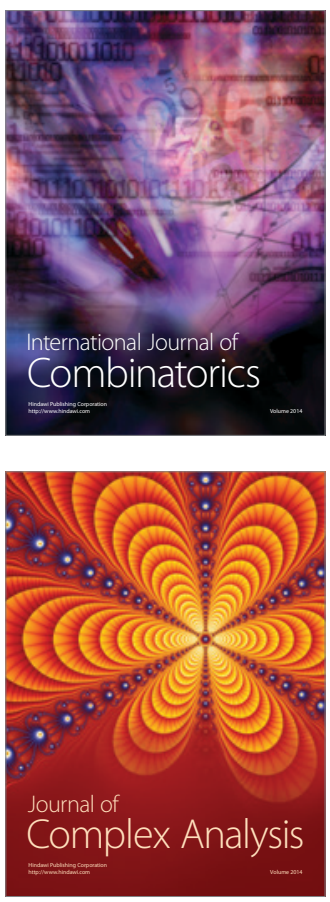

International Journal of

Mathematics and

Mathematical

Sciences
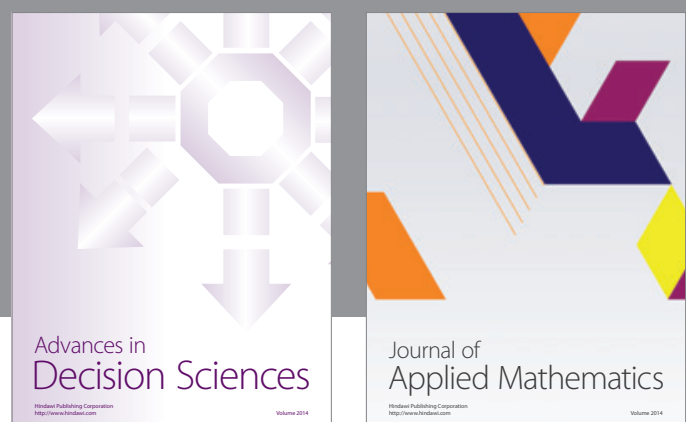

Journal of

Applied Mathematics
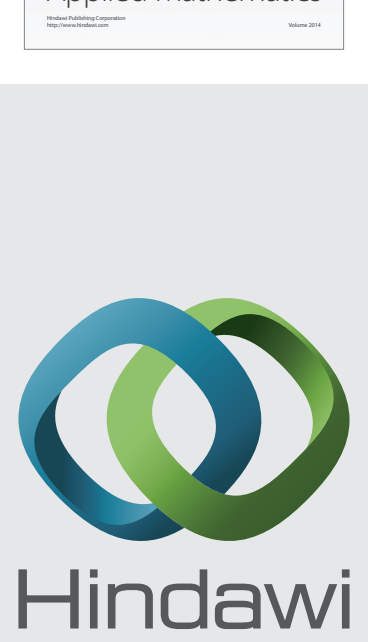

Submit your manuscripts at http://www.hindawi.com
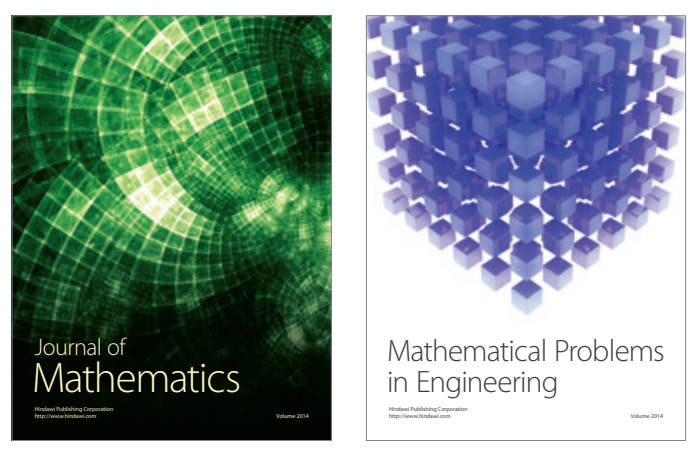

Mathematical Problems in Engineering
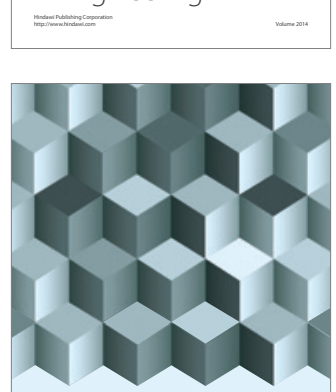

Journal of

Function Spaces
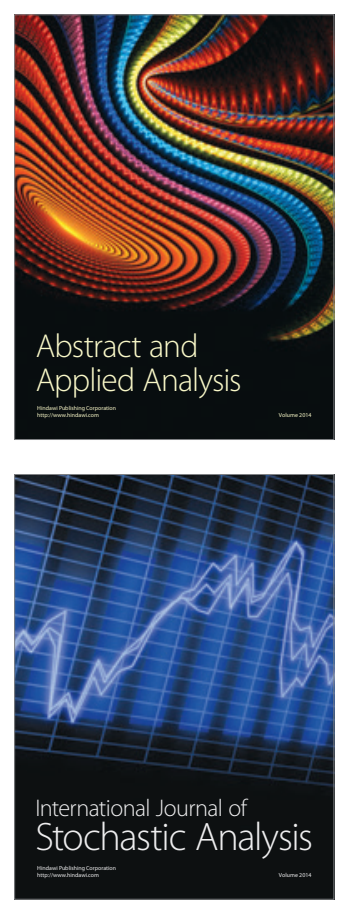

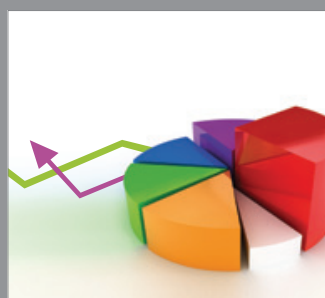

ournal of

Probability and Statistics

Promensencen
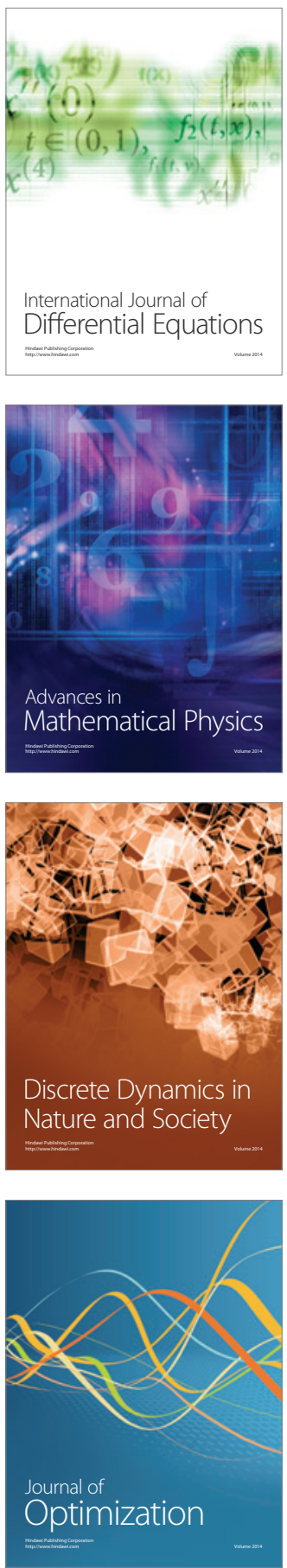